theme 4

technology

author(s) strand 2

materials, techniques and processes

\title{
Performing Human Pixels in Glitched: Portraits of Transformation
}

This paper examines the role of intentional error and unpredictable outcomes in digital image manipulation as a means of commenting on aspects of perfection, aesthetics, design process and technology. Glitched: Portraits of Transformation is a series of photographic human portraits that have been altered by performing JPEG images at the level of code bits. Intense bands and blocks of color, unorthodox textures, odd overlaps and crops - combined with machine-readable alphanumeric typography and philosophical quotes - are the result of a creative, collaborative design process. Informed by theories of 'research through design,' 'design authorship,' and 'failure studies,' the Glitched project operates at the intersection of representation, expression and intervention. While the results can be considered as artworks, the images address deeper concerns about the confluence of digitization and the human condition through design. The images, therefore, can be interpreted as visualizations of science's nascent field of 'synthetic biology' and its oncoming ethical and moral considerations. Additionally, the series represents the potential effects of physiological and behavior transformations. The designers begin with critical commentary on perfection in design followed by descriptions of their technical process in a theoretical context. Parallels of the Glitched series are drawn with the human condition, and the designers conclude with suggestions for viewer interpretation and further exploration.

keywords JPEG, glitch, portrait, perfection, digitization

Design often works towards an absolute of perfection: exquisite craftsmanship, high quality manufacturing, appropriately targeted function and ideal outcomes serve to propel design's progressive agenda. This notion is expressed within the design process by beginning at the divergent thinking and creating stage (lateral, exploratory, messy, ambiguous) and progressing through the latter convergent stage (refined, linear, focused and reductive). Digitization has furthered the expectations of perfection. Binary code is stable, infinitely reproducible and widely accessible through computing devices, resulting in the delivery - assuming equal hardware and software - of near perfect images, sounds and experiences on screens from Minneapolis to Milan to Mumbai.

This preoccupation with perfection can be found in modern industrial and information companies' manufacturing credo of Six Sigma. As a means to achieve nearperfect results, Six Sigma is a "disciplined, data-driven approach and methodology for eliminating defects (driving toward six standard deviations between the mean and the nearest specification limit) in any process - from manufacturing to transactional and from product to service." ('What is Six-Sigma' n.d.) Using Six Sigma's tenets, a company strives for a manufacturing rate of $99.99966 \%$ perfection, or no more than 3.4 imperfect parts 
theme 4

technology strand 2

materials, techniques and processes

per million. Design for Six Sigma (DFSS) extends this ideology into the design process, striving to design error out of the manufacturing and distribution equation. Similarly, revision control in software development provides a way to manage changes made over time to eliminate redundancies and conflicting errors in computer documents. Perfection, or as close to it as possible, is always the goal.

What, then, is the role of perfection's opposite (Shapley 2011: 5-20) in the design process - the mistake, the failure, the glitch? In communication theory, noise is typically perceived as having a negative impact on a signal - but when might the noise itself be more interesting, and perhaps more revelatory, than the signal? And, how might failures be used to investigate the designers' interests in identity and social issues? Examples of accidental technological glitching abound: out-of-focus photography, mis-registered fourcolor halftone screens, random 'make-ready' sheets in offset printing, slurred photocopies, poor television reception, decaying layers of billboards or posters, and more. The nature of digital images, however, invites human intervention at the level of pixels. Failures in digital media, accidental or not, have been celebrated in a variety of ways, such as online collections (for example, the Museum of Glitch Aesthetics), mobile apps developed to glitch user content (for example, KindleGlitcher) and various D.I.Y. tutorials.

Glitched: Portraits of Transformation is an experimental, collaborative design series that features photographic portraits corrupted with a palette of glitching techniques (Fig. 1). Each portrait consists of three elements: the original JPEG image (sourced primarily from the online photo sharing community Flickr, using Creative Commons share/remix licensing), the 'glitched' version of the image (created by manipulating the JPEG images at the level of code bits) and the image's alphanumeric code itself - incomprehensible to read, except by the computer - as a connecting typographic element. Colors used for the text and middle panels were sampled from within each image. Additionally, a quote relating to the concept of transformation is included in each; besides the selection of the original images and inevitable compositional decisions, the quote is the only deliberate authorial intervention in the triptych.

Figure 2. (authors of paper) (2013) Glitched: Portraits of Transformation entire series of twenty triptychs.

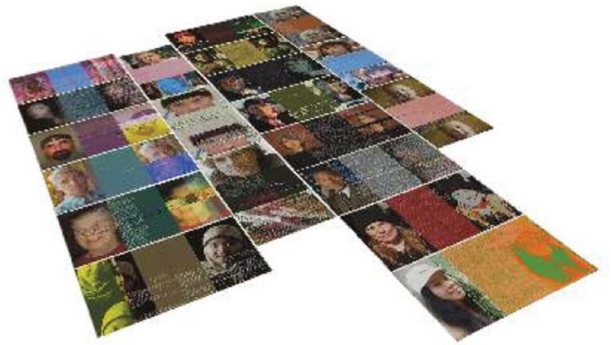

It is important to note two things about the images that were selected for Glitched. By perfection, the designers refer to the reproductive stability of each image's JPEG data, not to the physical appearance of the person depicted. Rather, people's faces were chosen for their diversity (age, expression, race, gender and so on); they represent the epitome of the human condition, not an ideal. The representational image is only 'perfect' to the degree that it is an objective likeness of the person being photographed. Because the images were sourced from Flickr, the designers have no knowledge of or subjective relationship with the subjects. 
William Mitchell uses the terms 'allographic' and 'autographic' in his book The Reconfigured Eye to suggest how artists might perform digital images (Mitchell 1992: 50-51). The terms are also explained in the article The Art Portrait, the Pixel and the Gene in the journal Convergence:

"Autographic refers to artforms that are unique, created in a single instance, and difficult to reproduce without degradation, like a painting. Allographic refers to artforms that are notational and performative, like sheet music or a theatrical script, original both in their conception and in their subsequent performance. Digital images, by Mitchell's definition, fit the allographic description. For example, an artist might create an image which could then be performed by other artists through manipulation of color, contrast, composition, cropping, applying special effect filters, adding collage elements, etc." (McCarthy 2005: 64)

In Glitched: Portraits of Transformation, the raw digital data of photographic images was interpreted as an allographic script; the designers 'performed' the JPEG code bits until a derivative image was produced. In Opera aperta, Umberto Eco describes an 'open work' as a work that is intentionally offered by a composer to a performer for completion through an interpretive dialogue (Eco 1989); the resulting work retains the character intended by the original author. While Eco introduces open work theory as it relates to musical scores, connections may be made in the context of a collaborative design process: one designer provides an image to the other designer for performance. The intention is not to reinvent the images, but rather augment digital representation through manipulation of an allographic script.

These digital images are open to errors, conflict and disruption as part of their performance. Isolated bits of digital data - as singular languages, media file types or alphanumeric sequences - are fragile homogenous entities; with one touch of interference from an outside source, the data changes and the pattern shifts. Adding, rearranging and deleting data will glitch digital files. It also means that two or more types of media can be combined; in Glitched, the designers brought together text and image at the level of code bits. Once a digital image's data is altered with text, the visual is transformed into something new and different.

As a computer-mediated performance, the tool of glitching is a central part of the message (Cascone 2000: 12-18). JPEG images, compressed using a 'lossy' compression algorithm to economize on file size, already have a compromised relationship to perfection. This was also the file type sourced from Flickr, the database for the original images. A JPEG's alphanumeric encoding, when opened in text editing software, follows the left-to-right and top-to-bottom order of writing systems derived from the Greek alphabet. Though unreadable by humans, this data can be shaped like any other body of text. An example of JPEG source code follows, as viewed within TextEdit software:

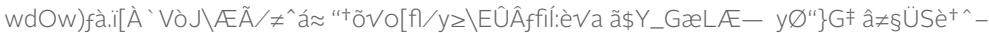

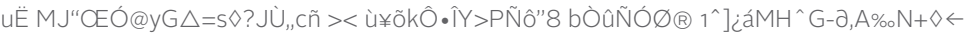

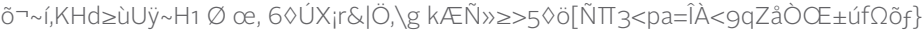
"€Õ《+)\%,æjÒ>ô\}

This technique for intentionally combining and altering media occupies a space alongside the practices of collage, montage and cut-up. The image was altered in the same horizontal direction as text, and rotating it 90 before glitching was used to introduce 
theme 4

technology

Figure 2.

(authors of paper) (2013) Glitched: Portraits of Transformation "Hui" triptych. strand 2

materials, techniques and processes

vertical effects. Single letters or numerals in the JPEG's source code were swapped or deleted, often resulting in thin bands of intense color (Fig. 2). The tools used were functions such as copy, cut, paste, find/change and delete; these actions led to unpredictable results that ranged from radical to subtle reconfigurations of the image. The designers' self-initiated brief adheres to the tenets of 'research through design,' because "in this way design researchers focus on making the right things, while design practitioners focus on making commercially successful things," [italics in original] (Zimmerman, Forlizzi \& Evenson 2007: 7) and design authorship, whereby an external client is unnecessary to commission the visual communication, allowing the designers to focus on th eir own content, meaning and audience (McCarthy 2013). Failure studies in design offer another model, particularly the writings of engineering scholar Henry Petroski (2006). The corporate design mantra 'fail faster' is a well known process to generate prototypes quickly, using failure to define success.

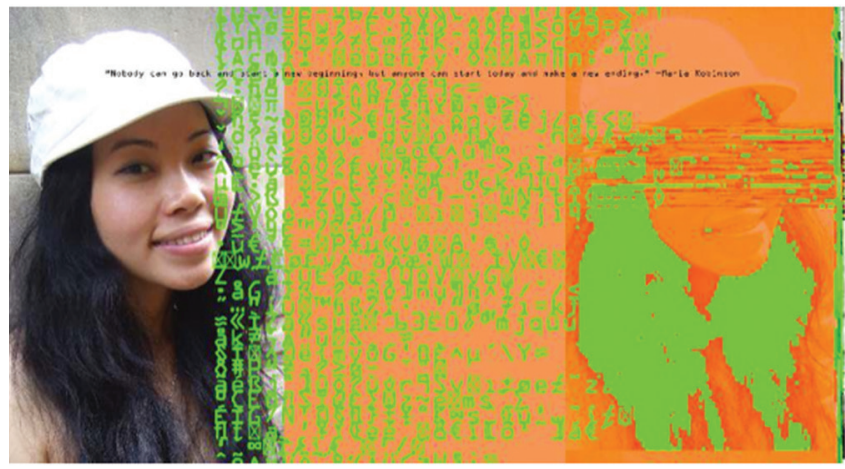

Through email, the designers openly exchanged digital files and invited the rearrangement of code-level data, intensity of personal expression and communicative use while preserving the character of the original image. Working collaboratively this way allowed for unplanned, serendipitous outcomes (Barker 2011); discussing the project through email and Skype separated written and verbal communication from the creative process itself. JPEG images were sourced from Flickr, and emailed to one another. The recipient glitched the image, then returned it to the sender. The sender then selected a quote to complement the photo, used JPEG data from the image as typography, sampled a color from the glitched version, and developed the triptych using the original and glitched images. For solo designers, there is a tendency to control and perfect all aspects of a product; with the two designers generating work asynchronously, however, the element of surprise became part of the process as digital files were downloaded and opened.

As a result of the designers' collaborative process, even their intentional 'glitching' interventions were subject to corruption as versions of images were created, shared and altered repeatedly. Files on one computer would sometimes open and appear differently from its sender. The synergies between the designers, images, technology and context in Glitched are of equal importance, and reflect the agency and activism required in the cyborg era (Inman 2004).

Performing a glitched image back and fourth turned "noise" artifacts into a type of communication code that is the absence of a message (Menkman 2011). The 
transformation of this glitched image as a non-message from onde designer to the other was subject to personal and social signals and gestures as the triptych was crafted into a final visual form. What is noise to one person is a message to another (Krapp 2011) and the designers assert that non-messages and noise hold communicative properties that can be used to investigate ideas beyond visual aesthetics. From code edits below the pixel surface to the visual manifestation above, these allographic scripts are performed first between software and designer, then as creative expression between two designers, and finally as a presentation to a viewer or audience. This process was repeated until a body of work was created that was cohesive yet visually diverse, and could illustrate the designers' inquiries on physiological and behavioral transformation.

The Glitched series suggests analogies in human portrait JPEGs with human physiology and behavior (Fig. 3). The act of 'damaging' faces may at first appear remote and detached, but viewers might consider the relationship between glitches (mistakes, accidents, juxtapositions, corruptions, etc.) as catalysts in affecting human change.

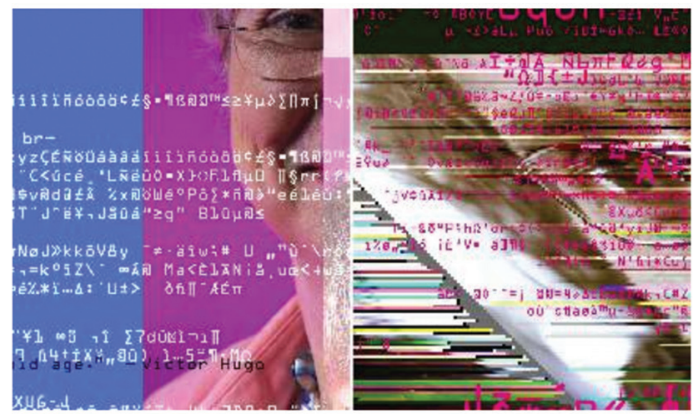

Similar to mistakes as 'diseases' in the development of digital images (Eiserman and Hushlak 2011: 93-102), human glitching exists in hormonal changes, disease and viruses through procreation and evolutionary mutations. Even aging itself may be viewed as the corruption of human 'software code' over time. Glitches to the human genome may result in cancers, growths and other medical conditions; approaching human representation as JPEG codes, the designers also draw connections to viruses and genetics. Scientist Craig Venter's research into using computation to design new organisms by manipulating their genetic code ('First Self-Replicating' 2010) has made digitization a potential tool of life-creation. The field of so-called 'synthetic biology' - a mix of biology and engineering promises technological innovations that might come with ethical and moral ramifications. In this context, glitched images seem less controversial, while glitched DNA might lead to Frankenstein-like aberrations.

Additionally, the triptychs represent the objective and subjective of human behavior in ways that can be perceived as both beautiful and ugly. Considering externally transformative influences such as education, persuasion, indoctrination, epiphany and any number of psychological factors, it could also be argued that behavior and appearance are governed by numerous glitches (inclusive of its positive connotations like serendipity and luck). Behavior that is subversive or irrational is usually viewed as corruptive, causing a breakdown - for better or worse - of society's acceptable norms. A glitch can change transformative genius into destructive madness. Conversely, behavior that is considered positive may be the harmonious fusion of oneself with external influences.
Figure 3 .

(authors of paper) (2013) Glitched: Portraits of Transformation details of Hayhurst and Zilverbat triptychs. 
Designed using techniques that simultaneously create and destroy, the mingling of accident and intent can alter perceptions of truth and fiction. This is revelatory in an examination of both design process and the human condition. Our biology and behavior, with all its failures and misgivings, has the capacity to manifest in ways that become attractive. These triptychs exemplify the curiosity and aesthetic appeal that accompanies breaking, failing and corrupting - and the tangible, compelling results. The designers also see opportunities in bringing their process and images into interactive digital media as a means to further investigate the possibilities of performing human pixels. Glitched: Portraits of Transformation is meant to challenge, delight - even 'glitch' - viewers' perceptions of what it may mean to be a photographically documented, and digitally performed, human.

To conclude, the value of this experiment is three-fold, like the triptychs themselves. Glitching the software code - making it imperfect - investigates the limits of coherent alphanumeric syntax of JPEG data. This enables image-makers to influence the image at the syntactic level, not just at the visual level (manipulation through Photoshop, for example). Two, the project conceptually addresses the potential convergence of human 'data' (genetic code) with computerized digitization. It speculates that synthetic biology will have moral and ethical implications in the near future, and also considers other external influences on behavior. And three, the Glitched triptychs can be interpreted aesthetically. Whether seen as ugly, beautiful, flawed, perfect or any range of impressions, the designed images are only complete with viewers' reactions.

\section{References}

Barker, T. (2011) 'Aesthetics of the Error: Media Art, the Machine, the Unforeseen and the Errant' in M. Nunes' (ed.) Error: Glitch, Noise and Jam in New Media Cultures. New York: Continuum, pp 42-58. Cascone, K. (2000) 'The Aesthetics of Failure: "Post-Digital" Tendencies in Contemporary Computer Music.' Computer Music Journal, 24(4), pp 12-18.

Eco, U. (1989) Opera aperta. Cambridge, MA: Harvard University Press.

Eiserman, J. and Hushlak, G. (2011) 'The Mistake: The Importance of Errors in Computergenerated Images.' The International Journal of the Image, 1(2), 93-102.

'First Self-Replicating Synthetic Bacterial Cell.' (2010) Retrieved November 4, 2013 from http:// www.jcvi.org/cms/research/projects/first-self-replicating-synthetic-bacterial-cell/overview/. Gaulon, B. (2012) KindleGlitcher. Retrieved March 25, 2014 from http://recyclism.com/ kindleglitcher.php Inman, J. (2004) Computers and Writing: The Cyborg Era. Maywah, NJ: Lawrence Erlbaum. Krapp, P. (2011) Noise Channels: Glitch and Error in Digital Culture. Minneapolis, MN: University of Minnesota Press. McCarthy, S. (2005) 'The Art Portrait, the Pixel and the Gene: Micro Construction of Macro Representation.' Convergence: The Journal of Research into New Media Technologies. Sage Publications, Vol. 11(4), 64 .

McCarthy, S. (2013) The Designer As... Author, Producer, Activist, Entrepreneur, Curator and Collaborator: New Models for Communicating. Amsterdam: BIS Publishers.

Menkman, R. (2011) The Glitch Moment(um). Amsterdam: Institute of Network Cultures. Mitchell, W. J. (1992) The Reconfigured Eye: Visual Truth in the Post-Photographic Era. Cambridge, MA: MIT Press.

Museum of Glitch Aesthetics (n.d.) Retrieved March 25, 2014 from http://www.glitchmuseum.com. Petroski, H. (2006) Success through Failure: the Paradox of Design. Princeton, NJ: Princeton University Press.

Shapley, G. (2011) 'After the Artefact: Post-Digital Photography in Our Post-Media Era.' Journal of Visual Art Practice 10(1), 5-20.

'What Is Six-Sigma?' (n.d.) Retrieved November 4, 2013 from http://www.isixsigma.com/new-tosix-sigma/getting-started/what-six-sigma/.

Zimmerman, J., Forlizzi, J., \& Evenson, S. (2007) 'Research Through Design as a Method for Interaction Design Research in HCl.' Human-Computer Interaction Institute. Paper 41. 